\section{Rodrigo Perez Fernandez Roberto Penas Lado}

http://dx.doi.org/10.21278/brod67404
ISSN 0007-215X

eISSN 1845-5859

\title{
HOW TO MANAGE SISTER SHIPS ON A SHIPBUILDING CAD TOOL. THE APPLICABILITY CONCEPT
}

UDC 629.5(05):629.5.081

Professional paper

\begin{abstract}
Summary
As a result of the increased pressure to reduce cost and delivery times of modern ships, many shipyards are revising their processes and tools to manage and share information across all shipyard departments. An important part of this process is in many cases the implementation of PLM (Product Lifecycle Management) Systems or an extended use of the PLM Systems to manage all the information that must be shared by the shipyard departments (engineering, purchasing, planning, operations, production, etc.). The solution presented in this paper for an advanced integration between the CAD and the PLM intends to comply with the most demanding requirements of the shipyards as well as to maintain the efficiency, the scalability and the performance of the shipbuilding CAD tool.

This paper presents in detail the architecture of the applicability solution as well as the expected advantages and benefits for any shipyard. The use of specialized shipbuilding CAD Systems in a shipbuilding environments is crucial for the efficient design and manufacturing of ships. The scalability refers to both the number of CAD users and to the number of vessel items to be handled. Vessels are very complex products that may be composed of millions of items, requiring a large number of designers, accessing concurrently to the vessel product model. The design cycles of these vessels are usually very long and there are many design changes along the whole vessel lifecycle. Performance is another critical requirement, especially in the detail design and manufacturing stages, when the detail design is almost complete, there are hundreds of users working on the model, model changes are constant and information for the production processes must be provided continuously.
\end{abstract}

Key words: $\quad$ Applicability, effectivity, PLM integration, shipbuilding software, ship design technology, CAD/CAM

\section{Introduction}

The use of specialized shipbuilding CAD Systems in marine environments is crucial for the efficient design and manufacturing of ships [2]. The heart of a shipbuilding CAD System as FORAN is a relational database (ORACLE) where the vessel CAD product model is stored. The product model includes geometry, topology, specialized technological and manufacturing information for all ship design disciplines and many relationships between the ship items. 
Shipbuilding CAD Systems working in marine environments offer significant advantages over other generic CAD applications, some of which can be relevant for the purpose of this paper:

- Specifically developed for shipbuilding.

- Availability of shipbuilding smart modelling tools.

- Incorporation of many years of shipbuilding knowledge.

- Outputs adapted to shipbuilding manufacturing processes.

- Proven scalability.

- Proven performance.

- Adapted to shipbuilding requirements [1].

- Reduction of design and manufacturing hours over generic CAD applications.

The main objective of this paper is to enable a shipbuilding CAD System, as FORAN, to support management of Ship Unit Series of projects. For this purpose, it is necessary to develop an application inside the CAD that it would be enhanced to allow the creation of a new ship series and the management of the projects included in it, representing the different vessel units as it is shown in figure 1.

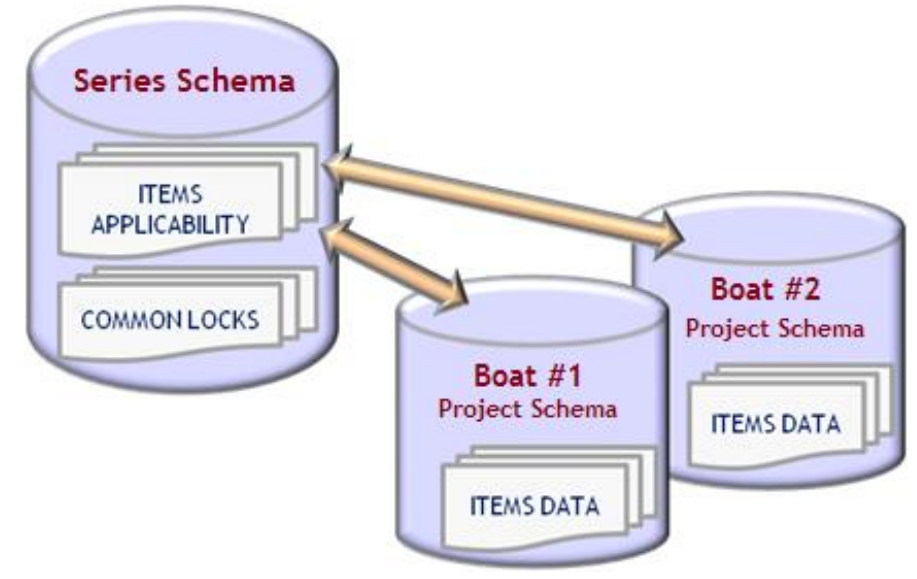

Fig. 1 CAD-PLM change process workflow

\section{Change and Configuration Management}

\subsection{Change Management}

A set of tools will exist in FORAN-PLM integration to handle the data involved in an ECN (Engineering Change Notice) process (see figure 2). Functionality is described below:

- ECN's as well as ECRs (Engineering Change Requests) will be managed in the PLM;

- When an entity is included in an ECN in either role (problem item, affected item or solution item), synchronization process in which the entity is included will hold data related to the $\mathrm{ECN}$;

- If a synchronized entity includes ECN data, the ECN will be registered in FORAN, if it is new;

- FORAN will have a ECN Manager tool to handle all data related to the ECN related to FORAN, which includes the status of the modifications expected in the change process for the entities; add additional entities to the ECN in either affected or solution entities, as detected in FORAN as a result of the work done or to be done in the model; and the execution status level of the ECN itself;

- A new filtering criterion will exist to select the entities related to an ECN, for publishing to PLM. 


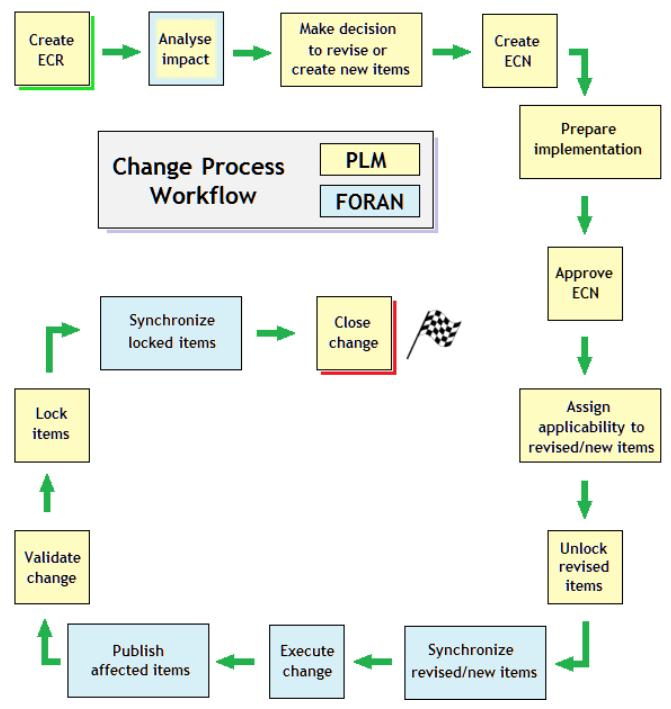

Fig. 2 FORAN-PLM as an example of a change process workflow

The "analyse impact" block in Figure 2 involves both CAD and PLM. This step is highly important due to a lot of decisions should be made at this point that they would impact downstream. Before to move to the next block "make decision to revise or create new items", any new ECN should be analysed from the CAD side and from the PLM side.

\subsection{Sister Vessels Managements}

This point mainly focuses in how to manage in the CAD-PLM integration the applicability of the items in a multi-vessel (sister ships) project context.

FORAN system already has functionality related to sister vessels management and therefore for the PLM integration some assumptions have been considered [5]:

- In a sister ships environment, each vessel or unit will be a FORAN project;

- There will exist a CLASS project to centralize FORAN locking and applicability data;

- The existence of an item in a FORAN project is considered as an item occurrence in that project;

- Modifications on an item will be done from a single project, but all other projects sharing the item with the same applicability will also be locked, to prevent modification on them by other user;

- Once the modification changes are applied, FORAN will update them in all projects sharing the items applicability (multi-save concept);

- The use of applicability on an item within a sister ship series might be:

$\circ$ (1) To all vessels (e.g., 1-UP).

○ (2) To all vessels newer than a specific one (e.g., 3-UP).

○ (3) To some vessels specifically (e.g., 2 and 6).

$\circ$ (4) A combination of the above (e.g., 1 and 4-UP).

If no applicability is set, it means "effective for all vessels" (1-UP). 


\subsection{Applicability Management in FORAN Modules}

Every project created belonging to a series of vessels will be uniquely identified with a specific vessel number (or unit number) allowing thus the mapping with the corresponding concepts in the PLM.

\subsection{Applicability Management in CAD-PLM Integration}

The necessary tasks required to gather information of the changes performed in PLM and to update the items in FORAN, through the CAD_PLM synchronization table, will be driven by one synchronisation process [4].

As the CAD-PLM synchronization table includes the applicability context information for each item, then every synchronization process will update the applicability data in the CLASS project.

If an applicability change is performed on an item in PLM, indicating a change in the range of units where the item is valid, that modification will be also updated in the FORAN projects accordingly, through the information stored in the CAD-PLM table as shown in figure 3 , where all the information is stored on the WBS (Work Breakdown Structure).

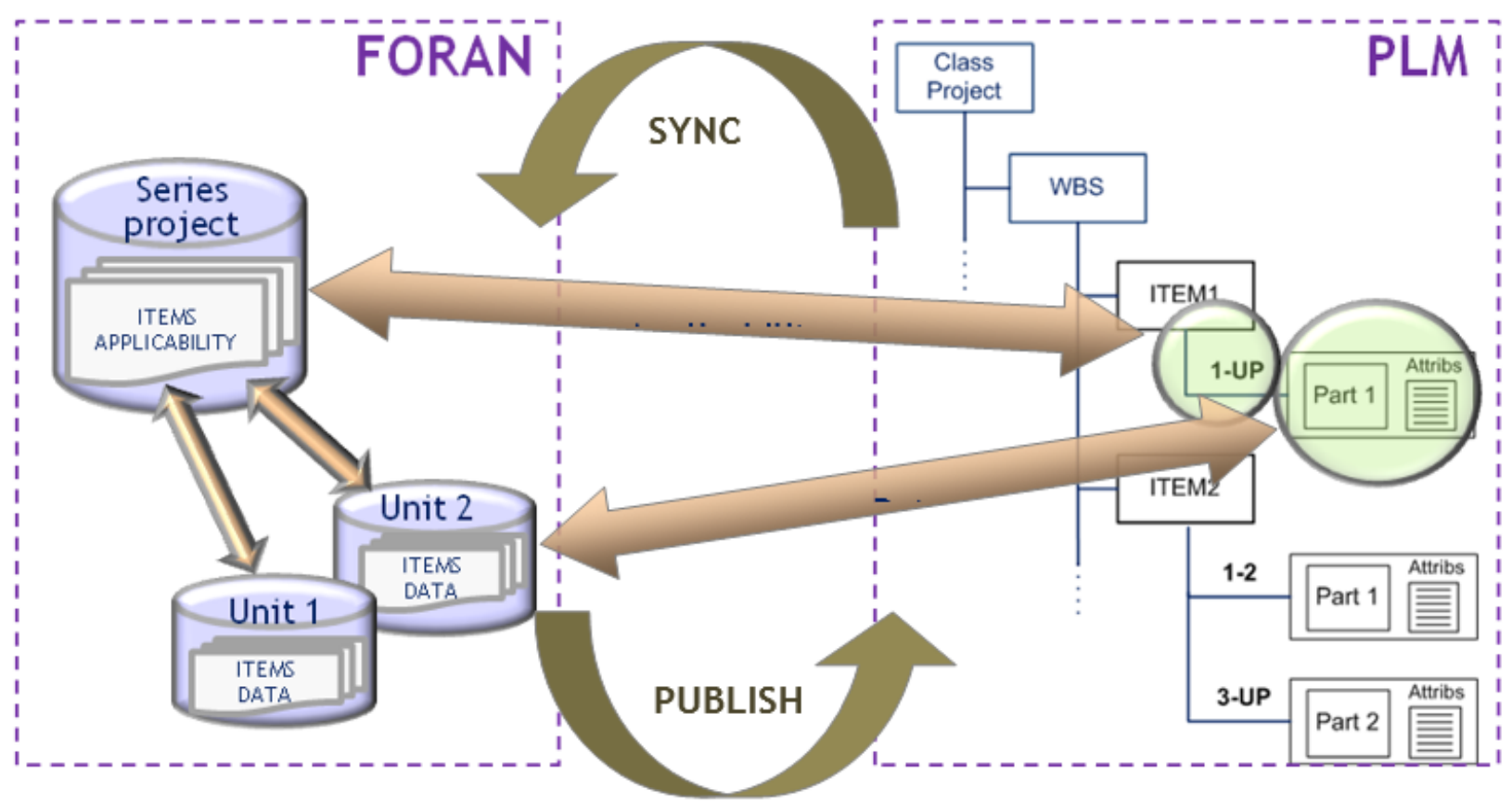

Fig. 3 Applicability management

For changing applicability range for an entity, the user must have the applicability manager role to be allowed to do so.

\subsubsection{Publish CAD Data to PLM in an Applicability Context}

Publishing CAD data to PLM, explained in figure 4, is subject to the following rules and features [3]:

- Normally, an applicability range is noted as 1 for the first vessel, 2 for the second, etc. According with this nomenclature, one change in the first vessel that will affect the rest of the series, it will noted as [1-UP]. A change just on the vessels second and third will be noted as [2-3]. And a change that it will affect from the third vessel, it will be shown as [3-UP]. 
- Items being published will include their applicability range ([2-UP], [2-4]...), so that the PLM integration layer will properly handle the data to publish in the appropriate way to support the configured effectivity context in PLM system.

- If an entity is included in more than one ship, the data will need to be published once for each applicability range. E.g., if an entity has two ranges: [1-2] and [3-UP], data will need to be published twice: one for [1-2] from either ship project \#1 or \#2 and another for [3-UP] from ship project \#3 or any other above.

- Publishing results to be stored in FORAN DB (where DB means Database) will be automatically propagated from the ship project being used to all others corresponding to the applicability range. E.g. publishing an item with range [1-3], if published from ship project \#3, results will be stored in ship \#3 project DB and will be automatically propagated to ships \#1 and \#2 databases without user interaction.

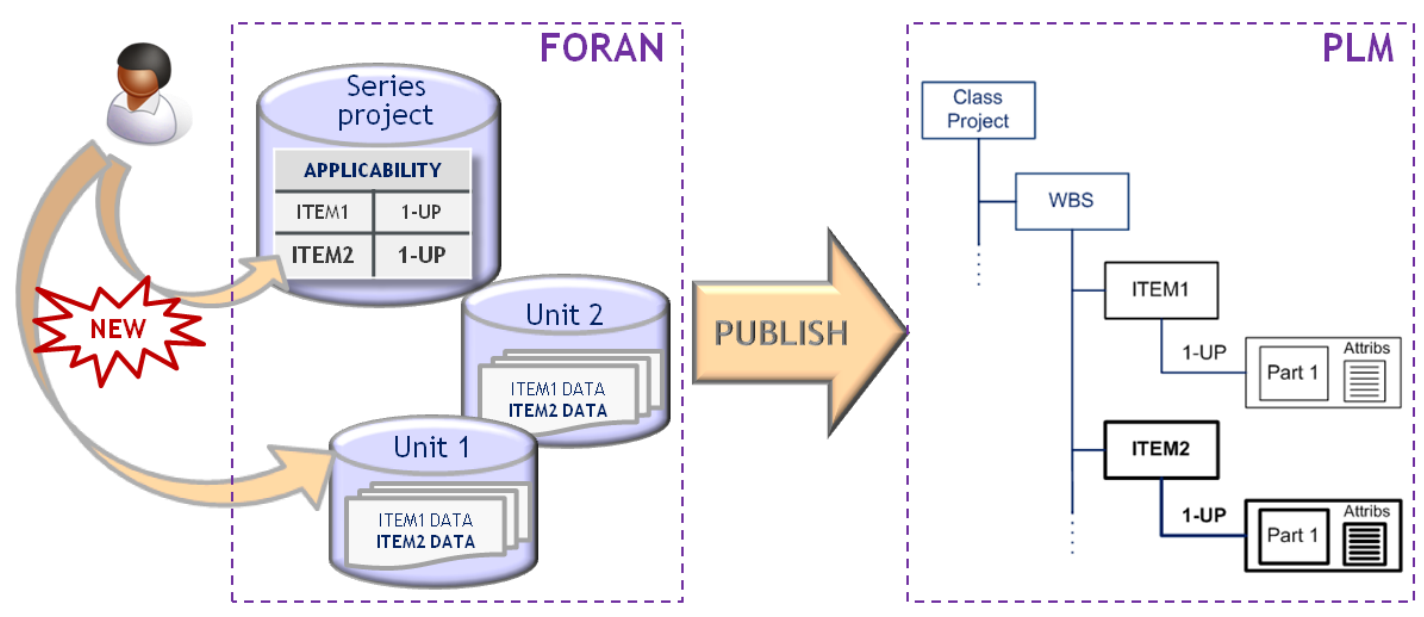

Fig. 4 Publish CAD data to PLM in an Applicability Context

\subsubsection{Synchronize Data from PLM in an Applicability Context}

Synchronizing data from PLM in an Applicability context is subject to the following rules and features:

- FORAN is considered master for establishing the applicability range values, so that they are propagated to PLM by publishing the entities.

- If a change in an applicability range (applicability cutback) is to be initiated in PLM, this must come as part of the changes specified in an ECN, so that changes will be performed in FORAN as indicated by that ECN.

- Items being synchronized will include their applicability range ([1-UP], [2-3], [3UP] ...), so that synchronization module (FSYNC) is capable of automatically choosing the appropriate FORAN project to apply the changes.

- Items being synchronized in a single process must include at least one common ship in their respective applicability ranges. E.g. a synchronization process with an item with [2-UP] and another one with [1-2] will choose ship FORAN project \#2 to apply synchronization changes.

- New items created in PLM must include the appropriate applicability value at synchronization, so that they could be finally created in FORAN with that applicability range. 
- Once the selected project for synchronization applies the changes, FORAN will propagate them to the appropriate FORAN projects in the series according to item applicability range in FORAN.

In the following image, figure 5, there is a data workflow showing the synchronization in applicability context.

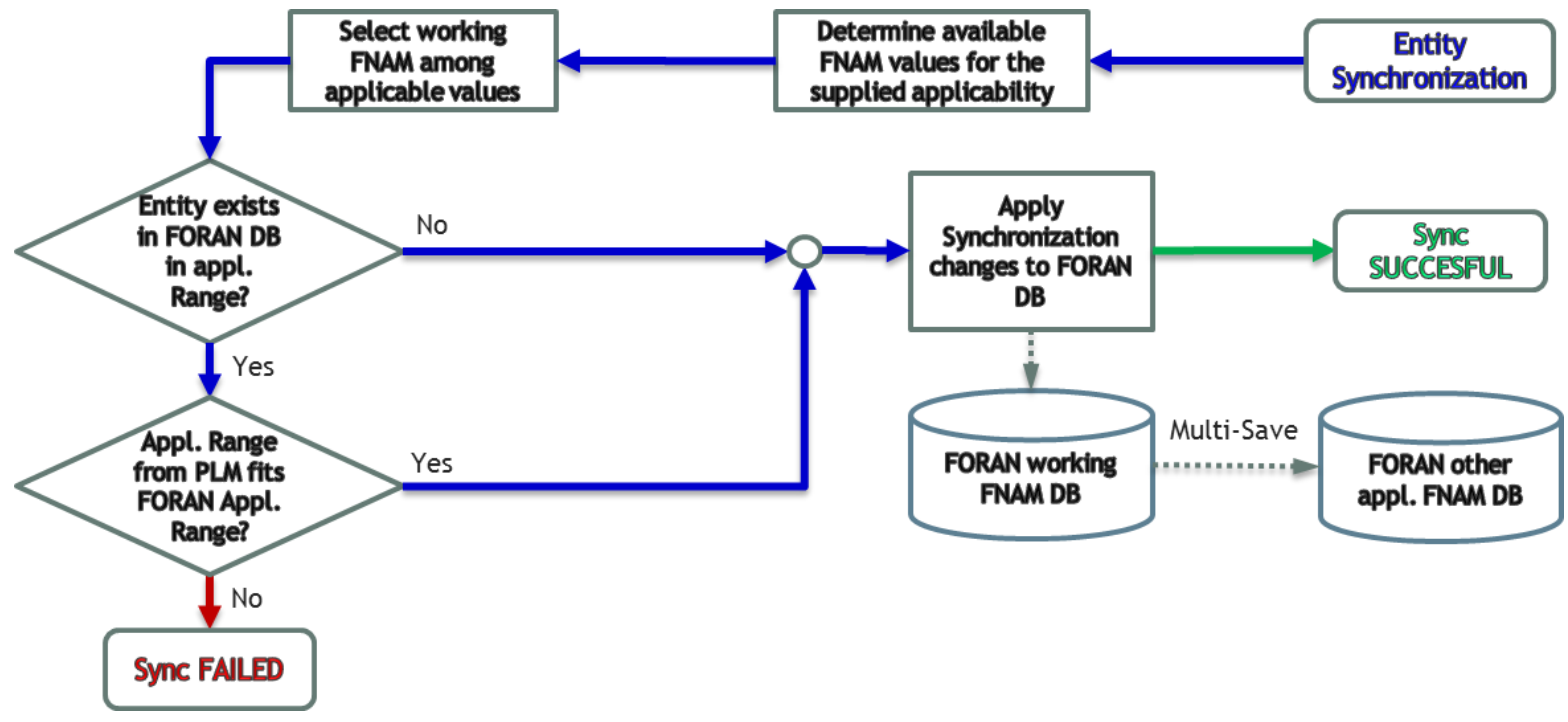

Fig. 5 Synchronization in Applicability context. Data workflow

It is also possible to show a schema of the synchronization data from PLM to the CAD System on figure 6 .

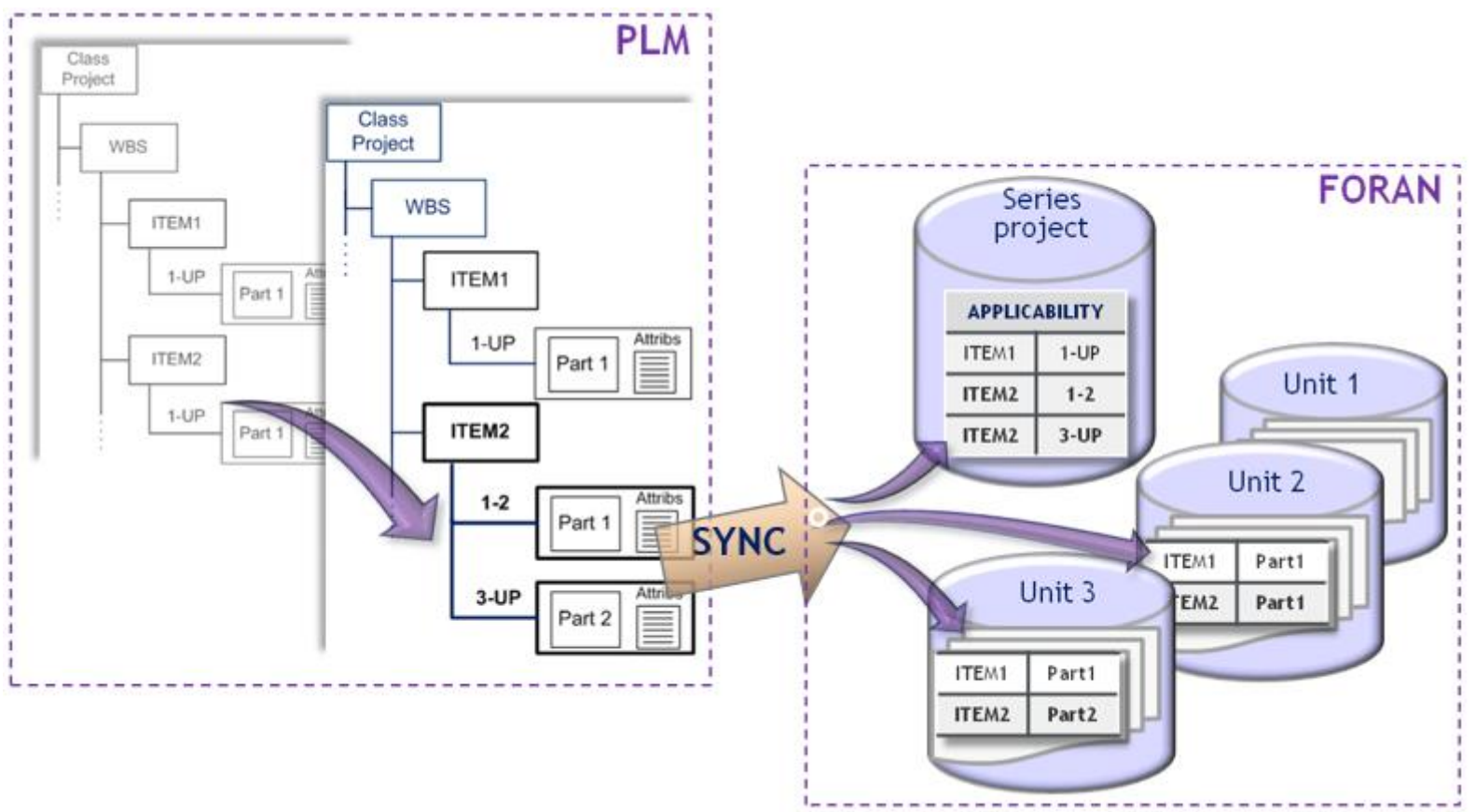

Fig. 6 Synchronizing data from PLM to FORAN 


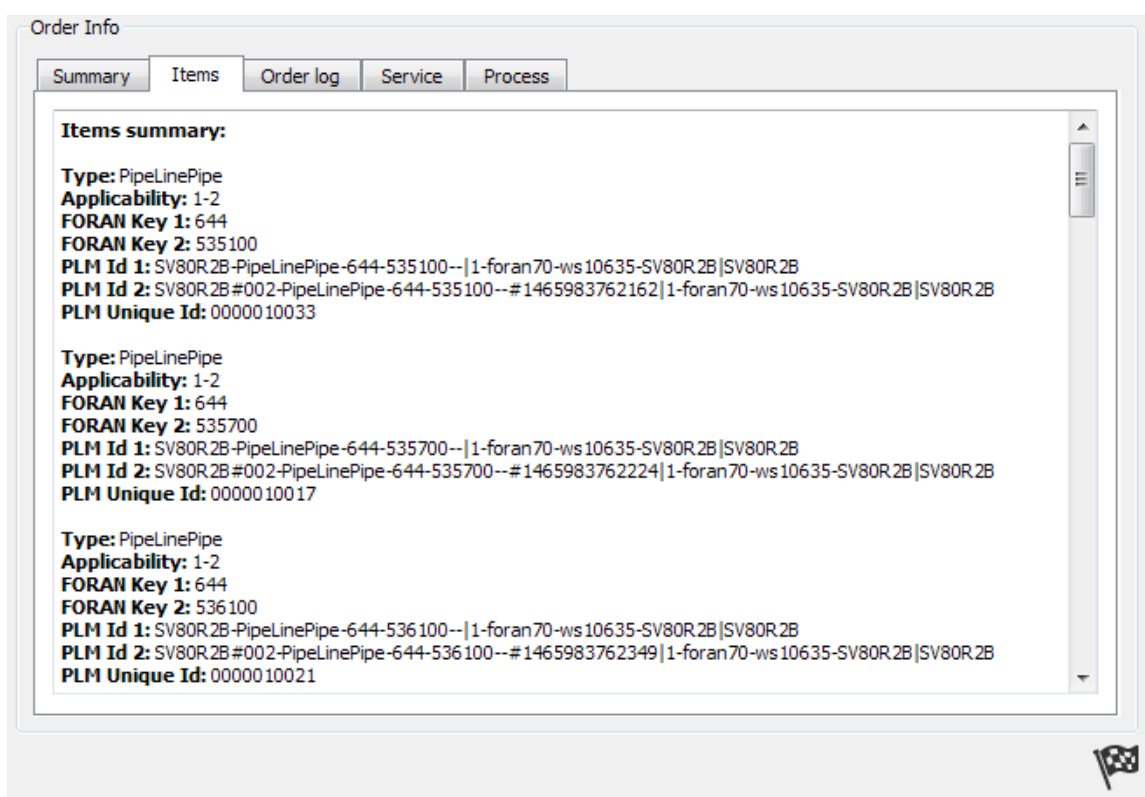

Fig. 7 List of pipe line items synchronized, with an applicability of [1-2]

\subsubsection{ECN Handling in an Applicability Context}

When handling ECN's in an applicability context, the following rules and features must be considered:

- ECN data is transferred from PLM to FORAN thorough synchronization mechanism.

- ECN's are suitable to include applicability data.

- The applicability range of an ECN specifies the range of ships in the series in which the ECN changes must be applied. Accordingly, the applicability range of the solution entities referenced in the ECN must match the applicability range of the ECN itself.

- The applicability range of entities referenced by an ECN as affected or impacted entities must include the range of the ECN applicability (see figure 8). This is because an ECN cannot reference an entity that is not applicable or does not exist in any of the ships to which the ECN changes must be applied.

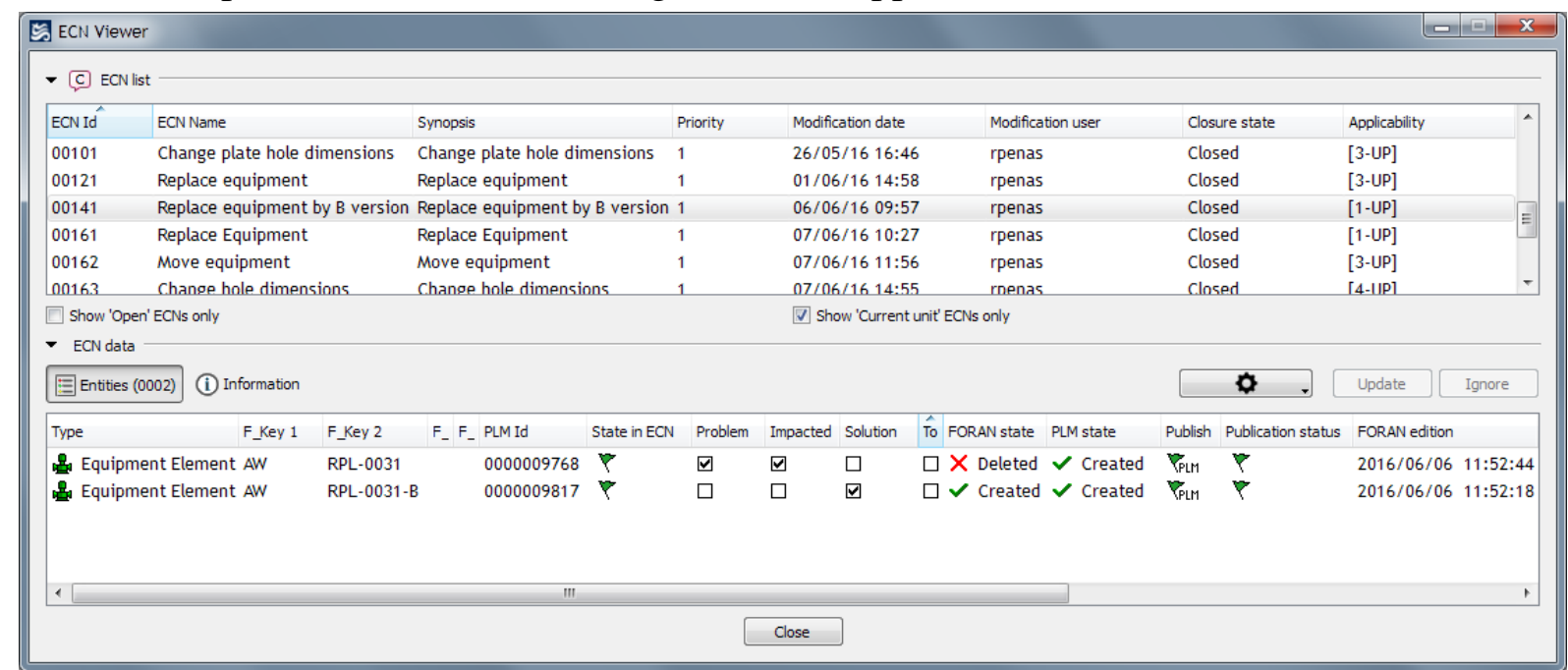

Fig. 8 Entities related to an ECN. Entity affected has been removed and solution entity has been created and published 
As an example, see figure 9, the following figure at the left will produce a successful synchronization, by applying synchronization to ship \#3. However, the example in the figure at the right will produce a failed synchronization, because applicability of ITEM 2 does not match any ship in the series to the ECN ([1-2] vs. [3-UP]), as well as the ITEM 1 item linked to the ECN does not cover the full range of the ECN applicability.
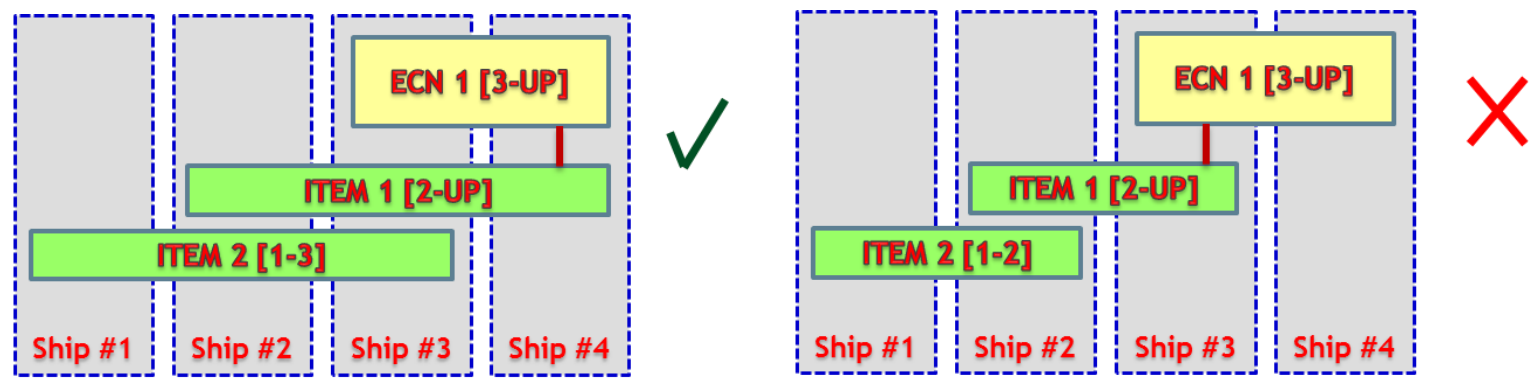

Fig. 9 Samples of success (left) and fail (right) synchronization of ECNs and items with applicability context. System will automatically select ship \#3 to apply synchronization changes

\subsection{Visualization of Differences between Ships in a Series}

A visualization tool exists to visually compare the differences of an entity between different ships (see figure 10).

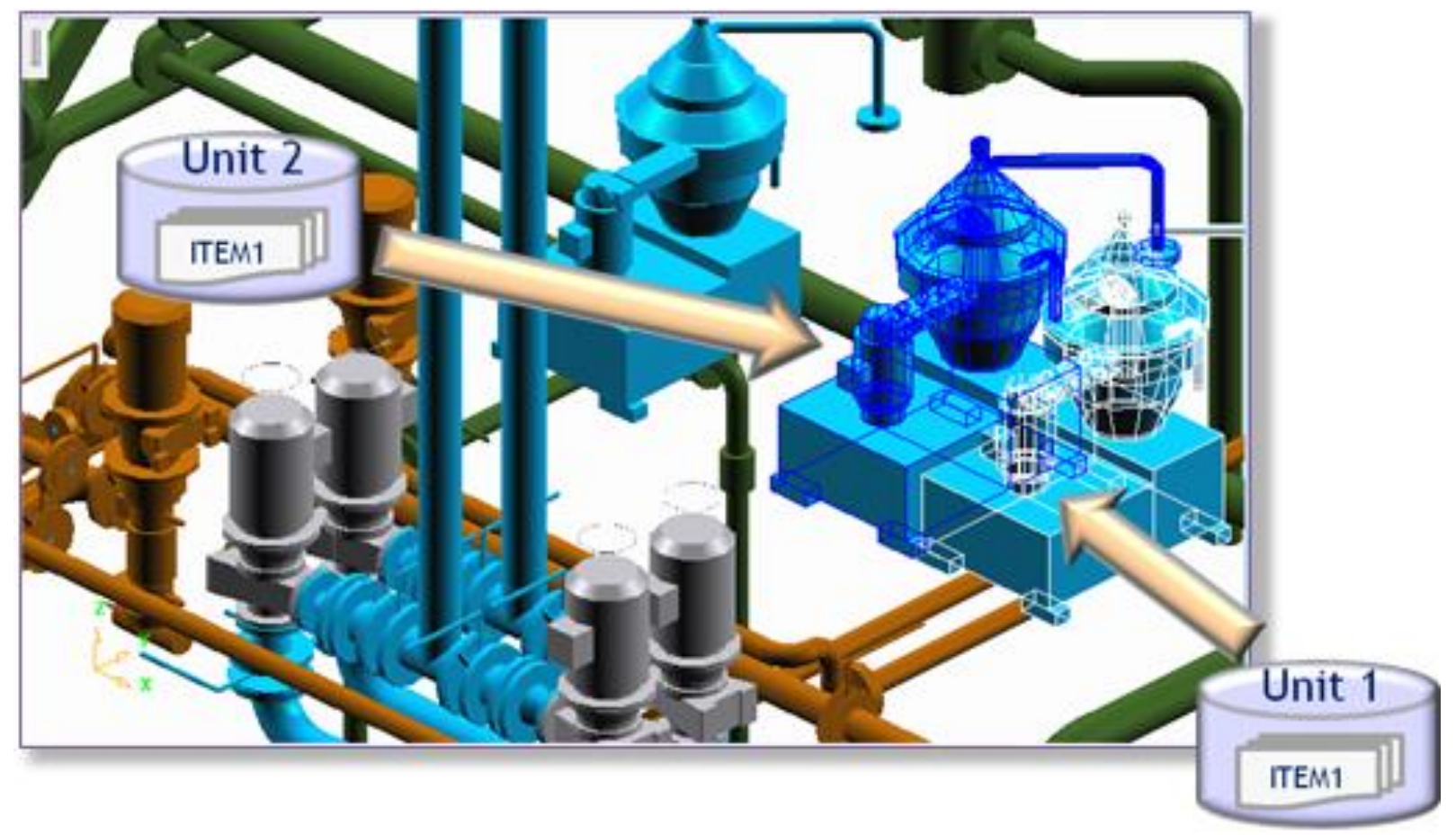

Fig. 10 Visual differences between an item in two series ship units

This tool allows comparing 3D graphic data as well as attributes, as it is shown below on figure 11 . 


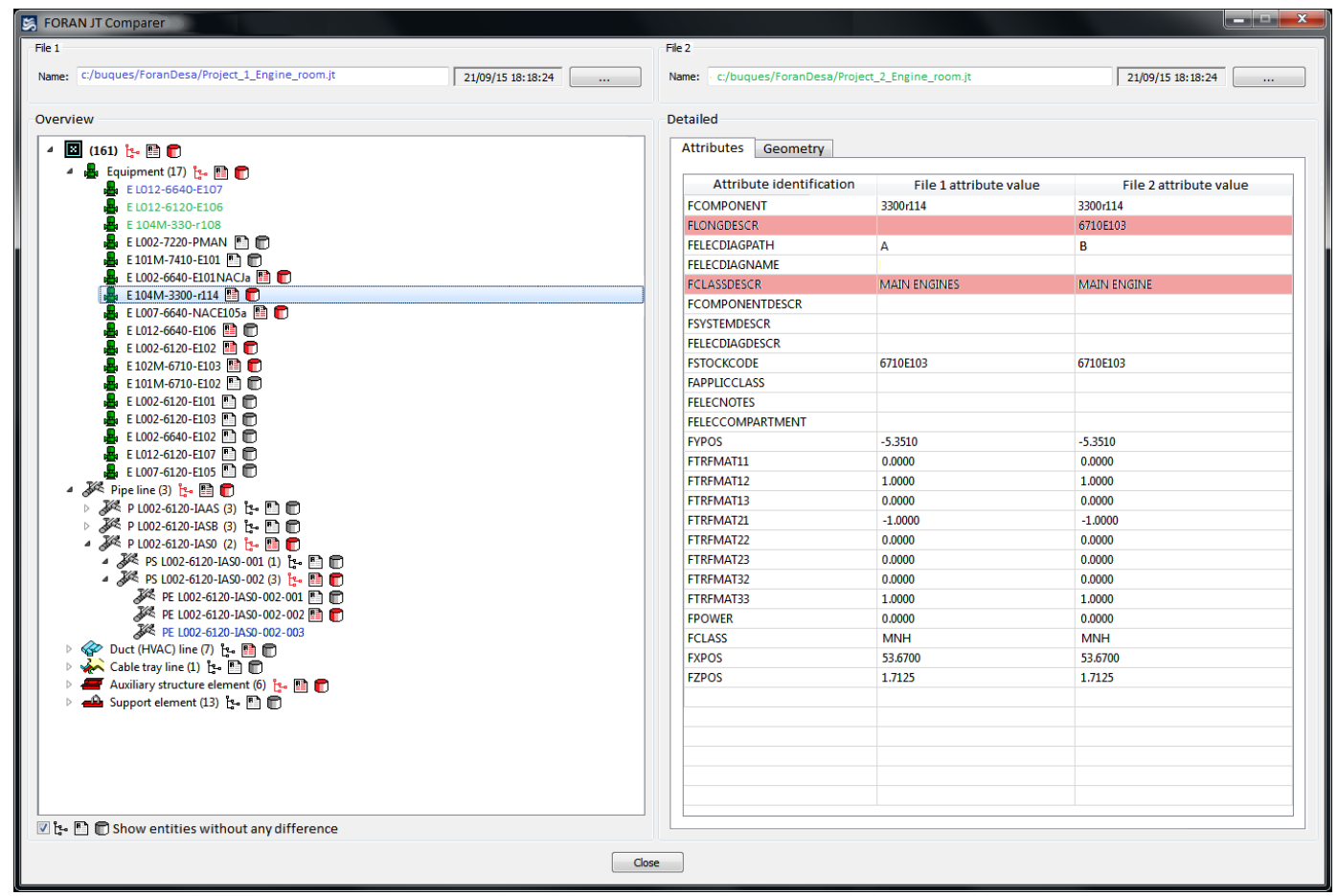

Fig. 11 Attribute comparison for an entity between two ships

It is also possible to show 3D graphics comparison between two ships for an entity, as it is shown on figure 12 .

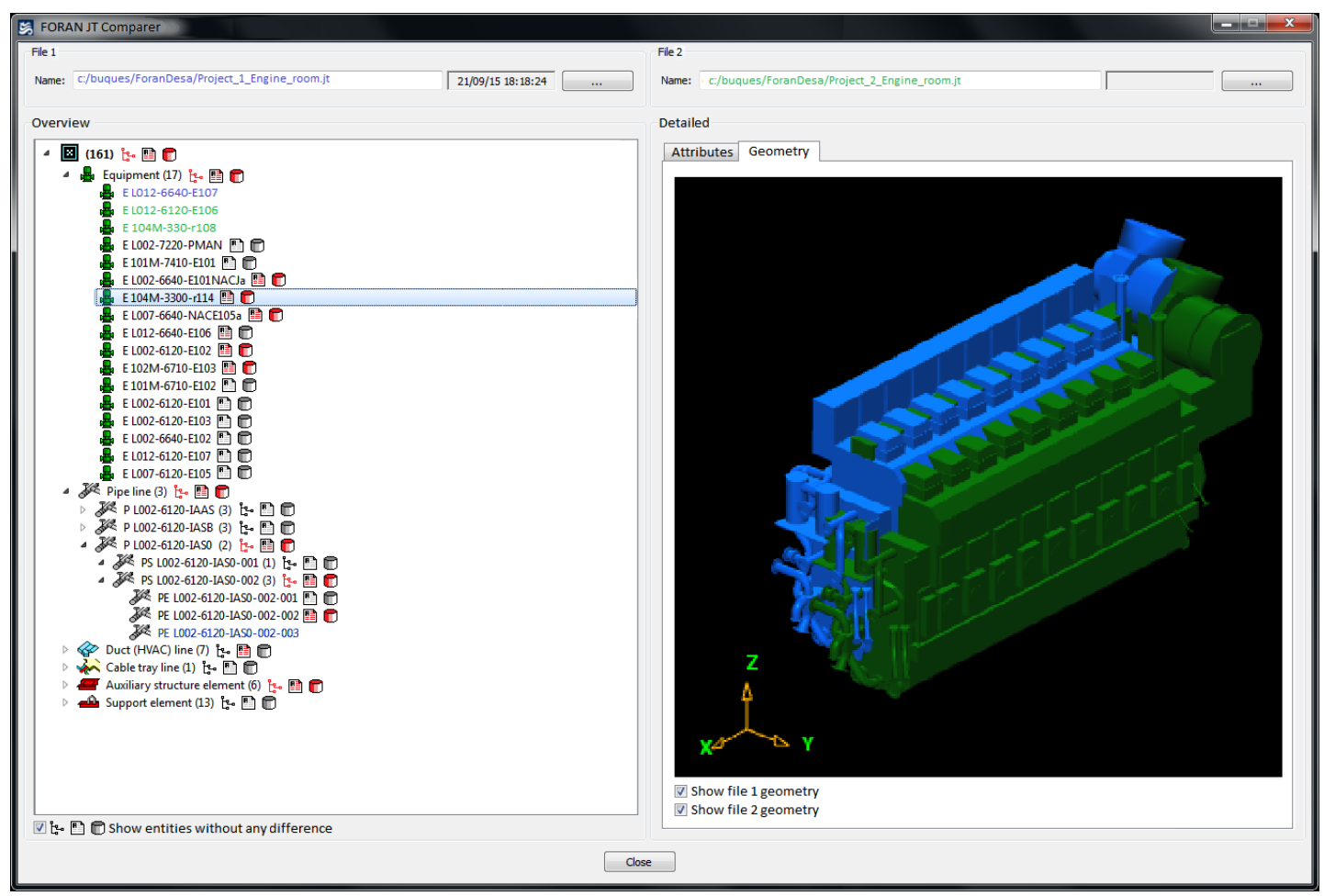

Fig. 12 3D graphics comparison between two ships for an entity 


\section{Conclusions}

This paper presents a solution for the applicability of a shipbuilding specific CAD System (FORAN) with an advanced PLM System in a Naval Shipbuilding environment.

As a result of the increased pressure to reduce cost and delivery times of modern ships, many shipyards are revising their processes and toolsets to optimize the management of sister ships. This paper describes how a strong CAD-PLM integration with efficient functionality on the CAD side, ensures a better design and production in a multi-vessel context. In order to address this complex challenge, and after having several years of experience in the development of the FORAN CAD/CAM System that is used in many important marine programs, SENER identified some remarkable assumptions to be considered:

- In a sister ships environment, each vessel or unit will have a CAD project.

- There will exist a Class project to centralize the CAD locking and applicability data.

- The existence of an item in the CAD project is considered as an item occurrence in that project.

Modifications on an item will be done from a single project, but all other projects sharing the item with the same applicability will also be locked, to prevent modification on them by other user. Once the modification changes are applied, the CAD will update them in all projects sharing the items applicability (multi-save concept).

The FORAN System already had functionality related to sister ships management in the past, but it has been notably improved with the complete FORAN-PLM integration. This paper presents in detail the architecture of the applicability solution as well as the expected advantages and benefits for the commercial and naval shipyards.

The proposed integration presents several important advantages:

- Takes profit of the experience and results of previous integration of FORAN with different PLM Systems.

- Incorporates the most outstanding requirements for the CAD - PLM integration coming from some relevant European shipbuilding companies, designing and manufacturing surface ships and submarines.

- Improves predictability by providing a single point of truth for the whole organization.

- Additionally, the design of the integration has been done with the objective of limiting the degree of coupling between the CAD and the PLM, with several important aims in mind:

- To reduce to a minimum the impact of the integration on the performance of both systems (the CAD and the PLM).

- To produce a scalable solution able to work with hundreds of designers in the CAD Engineering side and with thousands of PLM users in the whole shipbuilding organization.

- It would allow the PLM to take benefit of all the vessel information handled by the CAD from the early stages of the design.

The proposed integration is now under implementation for several important European Naval Shipbuilder. 


\section{REFERENCES}

[1] Perez, R. and Lee, D.-J. (2014). An innovative approach for Korean CADres. The Naval Architect Magazine. pp. 58-61.

[2] Dunseath, B.; Sear, C.; Murray, D. and McLauchlan, J. (2007). Choosing CAD tools for the $21^{\text {st }}$ Century. ICCAS 2007. Portsmouth. September.

[3] Penas, R.; Gomez, A.; Pastor, L. and Sanchez, L. (2009). A neutral framework for the integration of CAD in product model lifecycle systems. ICCAS 2009. Shanghai. September.

[4] Penas, R. and Gonzalez, C. (2011). Integration of DB oriented CAD systems with product lifecycle management. COMPIT 2011. Berlin. May.

[5] Pérez, R. and Penas, R. (2015). Integration between shipbuilding CAD Systems and a generic PLM tool in naval projects. Computer Science and Applications. Vol. 2. n. 5. pp. 181-191.

Submitted: $\quad$ 16.07.2016. Rodrigo Perez Fernandez, $\quad$ rodrigo.fernandez@ sener.es

Accepted: $\quad$ 07.09.2016. Marine Department. C/ Severo Ochoa, 4 - 28760 Tres Cantos - Madrid (Spain) 